\title{
PERSISTENCE AND UTILIZATION OF MATERNAL IRON FOR BLOOD FORMATION DURING INFANCY ${ }^{1,2}$
}

\author{
By CLEMENT A. SMITH, RUTH B. CHERRY, CONSTANTINE J. MALETSKOS, \\ JOHN G. GIBSON, 2ND, CHARLES C. ROBY, WILLIAM L. CATON, AND \\ DUNCAN E. REID
(From the Departments of Pediatrics, Medicine and Obstetrics, Harvard Medical School, The Radioactivity Center, Massachusetts Institute of Technology, and the Boston Lying-in Hospital, Children's Hospital and Peter Bent Brigham Hospital, Boston, Mass.)

(Submitted for publication March 21, 1955; accepted May 25, 1955)

The concentration of hemoglobin in the blood of normal infants falls progressively during the first 4 to 6 weeks following birth. The decline results primarily from reduced hematopoiesis, though iron is present for the manufacture of hemoglobin and replacement of erythrocytes. The slow rise in hemoglobin concentration which occurs during the remainder of infancy may be affected by several factors, including the rapidity of body growth, the amount and absorption of dietary iron, and the amount and conservation of iron which was obtained transplacentally (1). An opportunity to measure the persistence and utilization of transplacental iron during infancy arose as a by-product of investigations of maternal red cell volume and hematocrit recently published from this hospital $(2,3)$.

\section{MATERIAL AND METHODS}

In the original studies $(2,3)$, radioactive iron $\left(\mathrm{Fe}^{\mathrm{ss}}\right)$ prepared in the Massachusetts Institute of Technology cyclotron was administered as donor red cells to 11 women on four to seven occasions during pregnancy. From these 11 pregnancies and from five subsequent ones of four of the women, a total of 16 infants were available for study. The mothers and their infants were similar in diet, hygiene and general health to the random population of any obstetrical hospital or well-baby clinic in this area. Figure 1 shows the hemoglobin concentrations of the entire group of infants in comparison with controls from such clinics.

1 Some of these data were reported at the Annual Meeting, American Pediatric Society, French Lick, Indiana, May 6, 1950.

2 Supported in part by research grants from the Committee on Growth, American Cancer Society, to the Department of Pediatrics, Harvard Medical School, and from the National Institutes of Health to Harvard Medical School and the Massachusetts Institute of Technology; also by the joint program of the Office of Naval Research and the U. S. Atomic Energy Commission (H.M.S. and M.I.T.)
The total activity of $\mathrm{Fe}^{\mathrm{ss}}$ administered as tagged erythrocytes to each pregnant woman ranged from approximately 2 to 12 microcuries, equivalent to an initial dose rate of 0.1 to 1.0 millirad $^{3}$ per day in the mother's blood stream. The amount of radiation received by these infants was calculated according to the procedure described by Peacock, Evans, Irvine, Good, Kip, Weiss, and Gibson (5) on the basis of the experimentally measured radioactivity of the circulating hemoglobin iron. The cumulative radiation dose to the blood of the infants for calculation periods averaging 397 days (range 115 to 580 ) was an average of 131 millirads (range 30 to 354). The biological significance of these radiation levels is revealed by comparison with the cumulative dose to the blood stream received by the infants as a result of cosmic rays, terrestrial gamma rays and naturally occurring $\mathrm{K}^{+0}$ in the red cells, during the same observation periods. The total dose rate from these sources is about 0.5 millirad per day, and resulted in cumulative doses averaging 202 millirads (range 60 to 282). Thus the total radiation resulting from $\mathrm{Fe}^{\mathrm{s5}}$ was, on the average, less than that due to natural sources.

The first transfusion given in any pregnancy was at 230 days antepartum; the last was usually given within 10 days before delivery. The total of 400 to $700 \mathrm{ml}$. whole blood, transfused in units of $100 \mathrm{ml}$. over a period of four to seven months, was considered insufficient to distort the normal hematology of pregnancy. Seven to $15 \mathrm{ml}$. of maternal venous blood and cord blood were obtained at delivery, and similar amounts of the infants' venous blood obtained at varying ages thereafter. After heparinization, aliquots of each sample were used for R.B.C., W.B.C. and Hgb. determinations, total iron analyses and $\mathrm{Fe}^{\mathrm{s5}}$ measurements. All procedures followed standard practices. The $\mathrm{Fe}$ and $\mathrm{Fe}^{\mathrm{BS}}$ measurements were carried out by the methods described by Peacock, Evans, Irvine, Good, Kip, Weiss, and Gibson (5).

\section{RESULTS}

By the breakdown of the donor cells transfused during pregnancy, radioactive iron was released

3 The newly recommended, but still unofficial, unit of energy absorption is the $\mathrm{rad}=100$ ergs per $\mathrm{gm}$. One rad $=1.20 \mathrm{rep}_{83}=1.07 \mathrm{rep}_{93}$. 


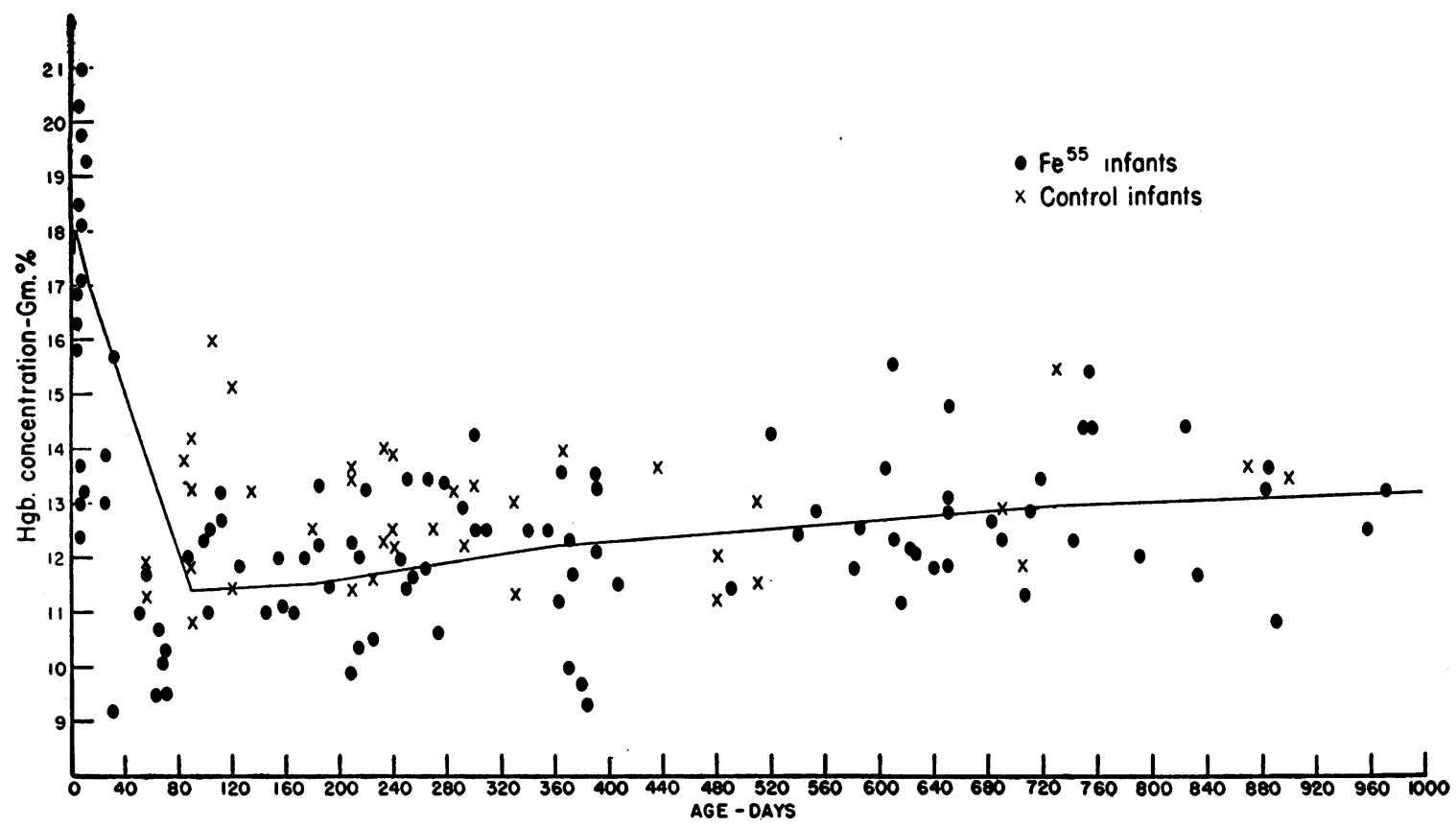

Fig. 1. Hemoglobin Concentrations of Infants Studied Compared to Controls of Similar Age

Controls consist of a group of normal infants, represented by X's (39 measurements on 33 infants), whose hemoglobins were measured concurrently with those of the infants whose blood contained $\mathrm{Fe}^{\mathrm{ss}}$. Line represents course of average normals from Diamond, Smith, and Vaughan (4).

into the general pool supplying the mother and fetus. Therefore, the blood of each infant at birth contained a measurable ratio of radioactive iron to packed red cells, from which the ratio of radioactive iron to hemoglobin and to hemoglobin iron was calculated. The latter ratio, referred to as specific activity, has been normalized to equal 100 per cent for each infant's cord blood sample. Postnatal changes in the specific activities are shown in Table I (Col. 10) and Figure 2. If each infant's hemoglobin had continued to be manufactured entirely from iron obtained transplacentally, all lines of the figure would have been horizontal at the 100 per cent level. The degree of downward trend with time is a measure of post-natally acquired iron used in blood formation. Thus, a specific activity of 75 per cent would indicate utilization of dietary iron for $100-75=25$ per cent of the infant's total circulating hemoglobin. ${ }^{4}$

4 Since the specific activity of only the circulating hemoglobin was determined and since several pools or compartments are involved in the dynamics of iron metabolism, constancy of circulating hemoglobin iron specific activity could have an alternate explanation. The alternative explanation would rest upon the assumption
The slight rise shown by the specific activities of blood samples obtained from several infants shortly after birth is presumably related to the timing of maternal iron administration. Since several transfusions of donor cells were given during the 11 pregnancies in which maternal hematology was under study, fetal hematopoiesis utilized iron with an increasing component of $\mathrm{Fe}^{55}$

that the fetus and infant had a compartment of stored iron with specific activity much higher than that of the circulating hemoglobin. Were this the case, dietary iron might replace original hemoglobin iron in the blood and the specific activity still be maintained constant by additional incorporation into hemoglobin of small, essentially negligible, amounts of iron from this special store. The fetuses, however, were supplied with iron which constantly contained $\mathrm{Fe}^{\mathrm{ss}}$. In 11 instances, $\mathrm{Fe}^{\mathrm{ss}}$ -was continuously present in the maternal blood during the latter two-thirds of gestation, although in slowly increasing amounts. In the other $5, \mathrm{Fe}^{\mathrm{ss}}$ was present in the maternal blood in a constant amount throughout the entire gestation period. In either case it would be expected that the specific activities of all the iron compartments in the body of any infant would be very nearly equal to each other at birth. The above assumption, then, would be unlikely and the alternative explanation improbable. 


\begin{tabular}{|c|c|c|c|c|c|c|c|c|c|c|c|c|c|}
\hline \multirow{2}{*}{$\begin{array}{l}(1) \\
\text { Infant } \\
\text { and } \\
\text { Specimen } \\
\text { number }\end{array}$} & \multirow{2}{*}{$\begin{array}{l}\text { (2) } \\
\text { Gesta. } \\
\text { age. } \\
\text { Sex, } \\
\text { etc. }\end{array}$} & \multirow[b]{2}{*}{$\begin{array}{c}(3) \\
\text { Age } \\
\text { (days) }\end{array}$} & \multirow[b]{2}{*}{$\begin{array}{l}(4) \\
\text { Wt. } \\
(K g .)\end{array}$} & \multirow[b]{2}{*}{$\begin{array}{c}(5) \\
\text { Length } \\
(\mathrm{cm} .)\end{array}$} & \multirow[b]{2}{*}{$\underset{(G m . \%)}{(6)}$} & \multirow[b]{2}{*}{$\begin{array}{l}\stackrel{(7)}{(7)} \\
\text { R.B.C. } \\
\text { (X106) }\end{array}$} & \multirow[b]{2}{*}{$\begin{array}{l}\text { (8) } \\
\text { Hct. } \\
(\%)\end{array}$} & \multirow[b]{2}{*}{$\begin{array}{c}(9)^{*} \\
\text { Cpm/ml. } \\
\text { packed } \\
\text { R.B.C. }\end{array}$} & \multirow{2}{*}{$\begin{array}{c}(10) \dagger \\
\text { "Normal- } \\
\text { ized" } \\
\text { specific } \\
\text { activity } \\
(\%)\end{array}$} & \multirow{2}{*}{$\begin{array}{c}(11) \ddagger \\
\text { Blood } \\
\text { vol. } \\
\text { (ml.) } \\
\text { (calc.) }\end{array}$} & \multicolumn{3}{|c|}{$\underset{\substack{(\text { gm.-calc. }) \\
\text { Circulating }}}{(14) \S}$} \\
\hline & & & & & & & & & & & Total & $\begin{array}{c}\text { From } \\
\text { transpl. } \\
\text { Fe }\end{array}$ & $\begin{array}{c}\text { From } \\
\text { dietary } \\
\text { Fe }\end{array}$ \\
\hline $\begin{array}{r}\text { 5GB-A } \\
\text { B } \\
\text { C } \\
\text { D } \\
\text { E } \\
\text { F } \\
\text { G } \\
\text { H } \\
\text { I } \\
\text { J }\end{array}$ & $\begin{array}{c}\sim 36 \text { wks. } \\
\sigma^{7}\end{array}$ & $\begin{array}{c}\text { Birth } \\
7 \\
65 \\
209 \\
309 \\
355 \\
616 \\
712 \\
884 \\
973\end{array}$ & $\begin{array}{r}2.53 \\
2.44 \\
5.45 \\
7.29 \\
8.37 \\
8.4 \\
9.7 \\
10.0 \\
11.1 \\
11.7\end{array}$ & $\begin{array}{l}49 \\
49 \\
55 \\
62 \\
67 \\
69 \\
77 \\
79 \\
83 \\
84\end{array}$ & $\begin{array}{l}20 \| \\
19.8 \\
10.7 \\
12.3 \\
12.5 \\
12.5 \\
11.2 \\
12.9 \\
13.2 \\
13.2\end{array}$ & $\begin{array}{l}6.2 \\
5.5 \\
3.3 \\
3.9 \\
6.0 \\
5.9 \\
4.2 \\
4.4 \\
4.8 \\
4.1\end{array}$ & $\begin{array}{l}54.3 \\
50.0 \\
31.7 \\
41.4 \\
38.3 \\
37.5 \\
35.7 \\
36.8 \\
39.7 \\
38.0\end{array}$ & $\begin{array}{r}185 \\
179 \\
143 \\
71 \\
82 \\
62 \\
52 \\
37 \\
31.4\end{array}$ & $\begin{array}{r}100 \\
90 \\
84 \\
47 \\
50 \\
39 \\
30 \\
22 \\
18\end{array}$ & $\begin{array}{l}208 \\
192 \\
374 \\
498 \\
575 \\
589 \\
701 \\
730 \\
806 \\
833\end{array}$ & $\begin{array}{r}41.6 \\
38.0 \\
40.0 \\
61.3 \\
71.9 \\
73.6 \\
78.5 \\
94.2 \\
106.4 \\
110.0\end{array}$ & $\begin{array}{l}41.6 \\
34.2 \\
33.6 \\
28.8 \\
36.0 \\
30.6 \\
28.3 \\
23.4 \\
19.8\end{array}$ & $\begin{array}{l}0 \\
3.8 \\
6.4 \\
32.5 \\
35.9 \\
\overline{47.9} \\
65.9 \\
83.0 \\
90.2\end{array}$ \\
\hline $\begin{array}{r}\text { 5GB2-A } \\
\text { B } \\
\text { C } \\
\text { D } \\
\text { E }\end{array}$ & $\begin{array}{c}\text { 38 wks. } \\
\text { Sister of } \\
5 G B \\
\&\end{array}$ & $\begin{array}{c}\text { Birth } \\
2 \\
9 \\
104 \\
193\end{array}$ & $\begin{array}{l}2.95 \\
2.72 \\
2.89 \\
5.56 \\
6.9\end{array}$ & $\begin{array}{l}47 \\
47 \\
47 \\
58 \\
60\end{array}$ & $\begin{array}{l}15.0 \\
16.9 \\
13.2 \\
12.5 \\
11.5\end{array}$ & $\begin{array}{l}4.4 \\
4.3 \\
3.5 \\
4.3 \\
4.2\end{array}$ & $\begin{array}{l}46.0 \\
46.5 \\
39.6 \\
37.2 \\
33.4\end{array}$ & $\begin{array}{r}93 \\
106 \\
92 \\
97 \\
74\end{array}$ & $\begin{array}{r}100 \\
102 \\
97 \\
101 \\
75\end{array}$ & $\begin{array}{l}232 \\
198 \\
207 \\
397 \\
461\end{array}$ & $\begin{array}{l}34.8 \\
33.5 \\
27.3 \\
49.6 \\
53.0\end{array}$ & $\begin{array}{l}34.8 \\
34.2 \\
26.5 \\
50.1 \\
39.8\end{array}$ & $\begin{array}{r}0 \\
-0.7 \\
0.8 \\
-0.5 \\
13.2\end{array}$ \\
\hline $\begin{array}{r}\text { 6GB-A } \\
\text { C } \\
\text { D } \\
\mathrm{E} \\
\mathrm{F} \\
\mathrm{G} \\
\mathrm{H} \\
\mathrm{I}\end{array}$ & $\begin{array}{c}\sim 38 \text { wks. } \\
\sigma^{2}\end{array}$ & $\begin{array}{c}\text { Birth } \\
12 \\
75 \\
245 \\
392 \\
644 \\
719 \\
959\end{array}$ & $\begin{array}{l}3.8 \\
3.77 \\
6.81 \\
10.5 \\
12.1 \\
14.2 \\
14.5 \\
16.0\end{array}$ & $\begin{array}{l}56 \\
56 \\
62 \\
74 \\
79 \\
88 \\
91 \\
95\end{array}$ & $\begin{array}{l}20 \| \\
19.3 \\
10.3 \\
12.0 \\
13.5 \\
12.9 \\
13.4 \\
12.5\end{array}$ & $\begin{array}{l}- \\
5.0 \\
3.3 \\
4.0 \\
5.1 \\
4.4 \\
4.9 \\
4.9\end{array}$ & $\begin{array}{l}65.7 \\
64.0 \\
30.9 \\
40.6 \\
41.6 \\
37.1 \\
37.5 \\
35.4\end{array}$ & $\begin{array}{l}62.8 \\
68.2 \\
70.3 \\
45.2 \\
41.6 \\
35 \\
33 \\
24\end{array}$ & $\begin{array}{r}100 \\
110 \\
103 \\
74 \\
62 \\
49 \\
45 \\
33\end{array}$ & $\begin{array}{r}332 \\
304 \\
479 \\
709 \\
802 \\
955 \\
994 \\
1,079\end{array}$ & $\begin{array}{r}66.4 \\
58.7 \\
49.3 \\
85.1 \\
108.3 \\
123.2 \\
133.2 \\
134.9\end{array}$ & $\begin{array}{l}66.4 \\
64.6 \\
50.8 \\
63.0 \\
67.1 \\
60.4 \\
59.9 \\
44.5\end{array}$ & $\begin{array}{c}0 \\
-5.9 \\
-1.5 \\
22.1 \\
41.2 \\
62.8 \\
73.3 \\
90.4\end{array}$ \\
\hline $\begin{array}{r}\text { 6GB2-A } \\
\text { B } \\
\text { C } \\
\text { D } \\
\text { E }\end{array}$ & $\begin{array}{c}40 \text { wks. } \\
\text { Brother } \\
\text { of } 6 \mathrm{~GB} \\
\sigma^{\top}\end{array}$ & $\begin{array}{c}\text { Birth } \\
6 \\
57 \\
112 \\
490\end{array}$ & $\begin{array}{c}3.70 \\
3.38 \\
4.99 \\
6.71 \\
12.3\end{array}$ & $\begin{array}{l}56 \\
56 \\
58 \\
63 \\
76\end{array}$ & $\begin{array}{l}18.2 \\
18.5 \\
11.7 \\
12.7 \\
11.5\end{array}$ & $\begin{array}{l}4.7 \\
5.1 \\
3.6 \\
3.7 \\
4.7\end{array}$ & $\begin{array}{l}56.1 \\
53.3 \\
30.1 \\
32.0 \\
36.0\end{array}$ & $\begin{array}{r}134 \\
145 \\
157 \\
162 \\
70\end{array}$ & $\begin{array}{r}100 \\
101 \\
98 \\
99 \\
53\end{array}$ & $\begin{array}{l}308 \\
283 \\
374 \\
479 \\
787\end{array}$ & $\begin{array}{l}56.1 \\
52.4 \\
43.8 \\
60.8 \\
90.5\end{array}$ & $\begin{array}{l}56.1 \\
52.9 \\
42.9 \\
60.2 \\
48.9\end{array}$ & $\begin{array}{r}0 \\
-0.5 \\
0.9 \\
0.6 \\
41.6\end{array}$ \\
\hline $\begin{array}{r}\text { 7GB-A } \\
\text { B } \\
\text { C } \\
\text { D } \\
\text { E } \\
\text { F } \\
\text { G } \\
\text { H }\end{array}$ & $\underset{\wp}{\sim 41 \text { wks. }}$ & $\begin{array}{c}\text { Birth } \\
2 \\
6 \\
25 \\
213 \\
374 \\
612 \\
793\end{array}$ & $\begin{array}{l}3.24 \\
2.95 \\
3.12 \\
3.52 \\
7.16 \\
8.6 \\
10.6 \\
11.4\end{array}$ & $\begin{array}{l}50 \\
50 \\
50 \\
54 \\
66 \\
74 \\
80 \\
87\end{array}$ & $\begin{array}{l}14.6 \\
14.3 \\
14.5 \\
13.0 \\
12.0 \\
11.7 \\
12.3 \\
12.5\end{array}$ & $\begin{array}{l}4.5 \\
4.5 \\
4.1 \\
4.0 \\
4.9 \\
4.2 \\
4.8\end{array}$ & $\begin{array}{l}40.4 \\
47.9 \\
49.3 \\
39.8 \\
41.6 \\
37.6 \\
33.8 \\
36.4\end{array}$ & $\begin{array}{l}93.5 \\
94.8 \\
85.8 \\
88.8 \\
81.0 \\
50.0 \\
26.7 \\
33.6\end{array}$ & $\begin{array}{r}100 \\
123 \\
113 \\
105 \\
109 \\
62 \\
28 \\
38\end{array}$ & $\begin{array}{l}248 \\
223 \\
233 \\
277 \\
521 \\
639 \\
761 \\
843\end{array}$ & $\begin{array}{r}36.2 \\
31.9 \\
33.8 \\
36.0 \\
62.5 \\
74.8 \\
93.6 \\
105.4\end{array}$ & $\begin{array}{l}36.2 \\
39.2 \\
38.2 \\
37.8 \\
68.1 \\
47.1 \\
26.2 \\
40.1\end{array}$ & $\begin{array}{c}0 \\
-7.3 \\
-4.4 \\
-1.8 \\
-5.6 \\
27.7 \\
67.4 \\
65.3\end{array}$ \\
\hline $\begin{array}{r}\text { 8GB-A } \\
\text { B } \\
\text { C } \\
\text { E } \\
\text { F } \\
\text { G } \\
\text { H }\end{array}$ & $\underset{\leftarrow}{\sim 38 \text { wks. }}$ & $\begin{array}{c}\text { Birth } \\
7 \\
36 \\
265 \\
367 \\
650 \\
825\end{array}$ & $\begin{array}{r}3.34 \\
3.04 \\
4.4 \\
8.8 \\
9.5 \\
11.9 \\
13.4\end{array}$ & $\begin{array}{l}52 \\
52 \\
57 \\
74 \\
78 \\
87 \\
93\end{array}$ & $\begin{array}{l}16.5 \| \\
17.1 \\
\overline{13.4} \\
13.5 \\
13.1 \\
14.4\end{array}$ & $\begin{array}{l}\overline{5.1} \\
4.5 \\
4.7 \\
4.6 \\
4.8\end{array}$ & $\begin{array}{l}44.6 \\
49: 7 \\
33.6 \\
43.4 \\
52.3 \\
40.7 \\
39.1\end{array}$ & $\begin{array}{l}277 \\
321 \\
332 \\
170 \\
128 \\
122 \\
116\end{array}$ & $\begin{array}{l}100 \\
124 \\
73 \\
66 \\
51 \\
42\end{array}$ & $\begin{array}{l}261 \\
242 \\
341 \\
643 \\
703 \\
868 \\
967\end{array}$ & $\begin{array}{r}43.1 \\
41.4 \\
-\overline{86.2} \\
94.9 \\
113.7 \\
139.2\end{array}$ & $\begin{array}{l}43.1 \\
51.3 \\
62.9 \\
62.6 \\
58.0 \\
58.5\end{array}$ & $\begin{array}{c}0 \\
-9.9 \\
23.3 \\
32.3 \\
55.7 \\
80.7\end{array}$ \\
\hline $\begin{array}{r}\text { 9GB-A } \\
\text { B } \\
\text { C } \\
\text { D } \\
\text { E } \\
\text { F } \\
\text { G }\end{array}$ & $\underset{q}{\sim 40 \text { wks. }}$ & $\begin{array}{c}\text { Birth } \\
2 \\
6 \\
197 \\
366 \\
623 \\
744\end{array}$ & $\begin{array}{c}3.02 \\
2.67 \\
2.87 \\
8.2 \\
10.2 \\
11.8 \\
13.0\end{array}$ & $\begin{array}{l}50 \\
50 \\
50 \\
66 \\
74 \\
80 \\
85\end{array}$ & $\begin{array}{l}19 \| \\
18.8 \\
16.7 \\
12.0 \\
12.5 \\
11.8 \\
12.3\end{array}$ & $\begin{array}{l}- \\
5.6 \\
5.0 \\
3.9 \\
4.8 \\
4.3 \\
4.6\end{array}$ & $\begin{array}{l}56.9 \\
51.4 \\
46.6 \\
42.2 \\
38.6 \\
33.6 \\
34.1\end{array}$ & $\begin{array}{l}537 \\
572 \\
510 \\
410 \\
364 \\
324 \\
241\end{array}$ & $\begin{array}{r}100 \\
97 \\
89 \\
90 \\
70 \\
57 \\
42\end{array}$ & $\begin{array}{l}252 \\
209 \\
221 \\
560 \\
692 \\
800 \\
885\end{array}$ & $\begin{array}{r}47.9 \\
39.3 \\
36.9 \\
67.2 \\
86.5 \\
94.4 \\
108.9\end{array}$ & $\begin{array}{l}47.9 \\
38.1 \\
32.8 \\
60.5 \\
60.6 \\
53.8 \\
45.7\end{array}$ & $\begin{array}{r}0 \\
1.2 \\
4.1 \\
6.7 \\
25.9 \\
40.6 \\
63.2\end{array}$ \\
\hline
\end{tabular}

* Observed $\mathrm{cpm} / \mathrm{ml}$. corrected for decay.

$\dagger \quad$ Specific activity at $\mathrm{t}=\mathrm{t}$

Specific activity at $\mathrm{t}=0$ (birth)

$\ddagger$ Blood volumes calculated from: A. Surface area by Du Bois and Du Bois (6) formula: S.A. $=$ Wt. $.^{0.425} \times$ L.0.72 $\times 71.84$; S.A. in sq. M., Wt. in Kg., L. in cm. B. Regression equation for volumes at birth calculated from data of Mollison, Veall, and Cutbush (7) on 28 normal infants: $y=58.9+0.456 \mathrm{X} ; \mathrm{y}=$ blood vol. in ml. $/ \mathrm{Kg}$., $\mathrm{X}=\mathrm{hct}$. in Col. 8 (this table) $\times 0.95$ (as correction for trapped plasma to conform with hcts. used by Mollison et al.). C. Regression equation for volumes after birth derived from data of Russell (8) on 40 normal infants and of Karlberg and Lind (9) on 64 normal infants: $y=1937 X-149 ; y=$ blood vol. in $\mathrm{ml} ., \mathrm{X}=\mathrm{X}$.A. in sq. $M$.

$\$$ For negative values in this column see comment in legend of Figure 4B.

\| Measurement not made; birth hgb. assumed from birth hct. and values from next blood sample. 


\begin{tabular}{|c|c|c|c|c|c|c|c|c|c|c|c|c|c|}
\hline \multirow{2}{*}{$\begin{array}{l}(1) \\
\text { Infant } \\
\text { and } \\
\text { Specimen } \\
\text { number }\end{array}$} & \multirow{2}{*}{$\begin{array}{l}\text { (2) } \\
\text { Gesta. } \\
\text { age. } \\
\text { Sex. } \\
\text { etc. }\end{array}$} & \multirow[b]{2}{*}{$\begin{array}{c}\text { (3) } \\
\text { Age } \\
(\text { days })\end{array}$} & \multirow[b]{2}{*}{$\begin{array}{l}(4) \\
\text { Wt. } \\
(K g .)\end{array}$} & \multirow[b]{2}{*}{$\begin{array}{l}(5) \\
\text { Length } \\
(\mathrm{cm} .)\end{array}$} & \multirow[b]{2}{*}{$\begin{array}{c}(6) \\
\mathrm{Hgb} \\
(\mathrm{Gm} . \%)\end{array}$} & \multirow[b]{2}{*}{$\begin{array}{l}\text { (7) } \\
\text { R.B.C. } \\
\left(\times 10^{6}\right)\end{array}$} & \multirow[b]{2}{*}{$\begin{array}{l}(8) \\
\text { Hct. } \\
(\%)\end{array}$} & \multirow[b]{2}{*}{$\begin{array}{l}(9) * \\
\text { Cpm/ml. } \\
\text { packed } \\
\text { R.B.C. }\end{array}$} & \multirow{2}{*}{$\begin{array}{l}(10) \dagger \\
\text { "Normal- } \\
\text { ized" } \\
\text { specific } \\
\text { activity } \\
(\%)\end{array}$} & \multirow{2}{*}{$\begin{array}{l}(11) \ddagger \\
\text { Blood } \\
\text { vol. } \\
(m l .) \\
\text { (calc.) }\end{array}$} & \multicolumn{3}{|c|}{$\underset{\substack{(12) \\
\text { Circulating Hgb. } \\
(\text { gm.-calc. })}}{(13) \S}$} \\
\hline & & & & & & & & & & & Total ${ }^{t}$ & $\underset{\substack{\text { From } \\
\text { transpl. } \\
\mathrm{Fe}}}{ }$ & $\begin{array}{c}\text { From } \\
\text { dietary } \\
\mathrm{Fe}\end{array}$ \\
\hline $\begin{array}{r}11 \mathrm{~GB}-\mathrm{A} \\
\mathrm{B} \\
\mathrm{C} \\
\mathrm{D}\end{array}$ & $\begin{array}{c}\sim 38 \text { wks. } \\
\sigma^{7}\end{array}$ & $\begin{array}{c}\text { Birth } \\
7 \\
64 \\
216\end{array}$ & $\begin{array}{l}2.77 \\
2.64 \\
5.27 \\
9.7\end{array}$ & $\begin{array}{l}47 \\
47 \\
57 \\
72\end{array}$ & $\begin{array}{r}16.8 \\
13.0 \\
9.5 \\
13.2\end{array}$ & $\begin{array}{l}5.1 \\
4.0 \\
3.3 \\
4.2\end{array}$ & $\begin{array}{l}48 \\
35.5 \\
29.6 \\
41.2\end{array}$ & $\begin{array}{l}42.3 \\
44.5 \\
41.5 \\
27.0\end{array}$ & $\begin{array}{r}100 \\
100 \\
107 \\
70\end{array}$ & $\begin{array}{l}221 \\
194 \\
380 \\
663\end{array}$ & $\begin{array}{l}37.1 \\
25.2 \\
36.1 \\
87.5\end{array}$ & $\begin{array}{l}37.1 \\
25.2 \\
38.6 \\
60.4\end{array}$ & $\begin{array}{c}0 \\
0 \\
-2.5 \\
27.1\end{array}$ \\
\hline $\begin{array}{r}12 \mathrm{~GB}-\mathrm{A} \\
\mathrm{B} \\
\mathrm{C} \\
\mathrm{D} \\
\mathrm{E} \\
\mathrm{F} \\
\mathrm{G} \\
\mathrm{H}\end{array}$ & $\underset{\sigma^{7}}{\sim 40 \mathrm{wks} .}$ & $\begin{array}{c}\text { Birth } \\
2 \\
6 \\
127 \\
225 \\
372 \\
541 \\
757\end{array}$ & $\begin{array}{c}3.61 \\
3.26 \\
3.44 \\
7.21 \\
8.4 \\
9.8 \\
10.9 \\
11.8\end{array}$ & $\begin{array}{l}49 \\
49 \\
49 \\
65 \\
70 \\
74 \\
80 \\
87\end{array}$ & $\begin{array}{l}20.5 \\
15.8 \\
13.7 \\
11.9 \\
13.3 \\
10.0 \\
12.4 \\
14.4\end{array}$ & $\begin{array}{l}6.2 \\
5.0 \\
4.2 \\
3.8 \\
5.8 \\
4.8 \\
5.2 \\
4.7\end{array}$ & $\begin{array}{l}67.4 \\
49.5 \\
51.4 \\
38.2 \\
39.0 \\
36.9 \\
38.6 \\
39.4\end{array}$ & $\begin{array}{l}267 \\
362 \\
296 \\
232 \\
260 \\
198 \\
147\end{array}$ & $\begin{array}{r}100 \\
129 \\
126 \\
85 \\
109 \\
70 \\
46\end{array}$ & $\begin{array}{l}318 \\
236 \\
246 \\
515 \\
601 \\
676 \\
771 \\
864\end{array}$ & $\begin{array}{r}65.2 \\
37.3 \\
33.7 \\
61.3 \\
79.9 \\
67.6 \\
95.6 \\
124.4\end{array}$ & $\begin{array}{l}65.2 \\
48.1 \\
42.5 \\
52.1 \\
73.7 \\
66.9 \\
57.2\end{array}$ & $\begin{array}{r}0 \\
-10.8 \\
-8.8 \\
9.2 \\
-\overline{6} \\
28.1 \\
67.7\end{array}$ \\
\hline $\begin{array}{r}2 \mathrm{~GB} 2-\mathrm{A} \\
\mathrm{B}\end{array}$ & $\begin{array}{c}\sim 40 \text { wks. } \\
\text { Sister of } \\
12 \mathrm{~GB} \\
\&\end{array}$ & $\begin{array}{c}\text { Birth } \\
\quad 7\end{array}$ & $\begin{array}{l}3.66 \\
3.46\end{array}$ & $\begin{array}{l}53 \\
53\end{array}$ & $\begin{array}{l}16.9 \\
17.8\end{array}$ & $\begin{array}{l}4.6 \\
5.1\end{array}$ & $\begin{array}{l}56.0 \\
57.6\end{array}$ & $\begin{array}{l}143 \\
146\end{array}$ & $\begin{array}{l}100 \\
100\end{array}$ & $\begin{array}{l}305 \\
269\end{array}$ & $\begin{array}{l}51.5 \\
47.9\end{array}$ & $\begin{array}{l}51.5 \\
47.9\end{array}$ & $\begin{array}{l}0 \\
0\end{array}$ \\
\hline $\begin{array}{r}\text { 13GB-A } \\
\mathrm{B} \\
\mathrm{C} \\
\mathrm{D} \\
\mathrm{E} \\
\mathrm{F} \\
\mathrm{G}\end{array}$ & $\begin{array}{l}\sim 39 \text { wks. } \\
\underset{\odot}{ }\end{array}$ & $\begin{array}{c}\text { Birth } \\
2 \\
6 \\
70 \\
153 \\
373 \\
526\end{array}$ & $\begin{array}{l}3.2 \\
2.86 \\
2.92 \\
5.1 \\
7.4 \\
10.0 \\
12.1\end{array}$ & $\begin{array}{l}45 \\
45 \\
45 \\
59 \\
66 \\
78 \\
83\end{array}$ & $\begin{array}{r}20.3 \\
12.9 \\
12.4 \\
9.5 \\
12.0 \\
12.3 \\
14.2\end{array}$ & $\begin{array}{r}5.0 \\
4.2 \\
3.9 \\
.3 .6 \\
4.0 \\
4.6 \\
4.4\end{array}$ & $\begin{array}{l}58.4 \\
40.4 \\
35.5 \\
27.8 \\
38.3 \\
35.3 \\
38.8\end{array}$ & $\begin{array}{r}156 \\
160 \\
189 \\
177 \\
123 \\
76\end{array}$ & $\begin{array}{l}100 \\
111 \\
120 \\
115 \\
79 \\
46\end{array}$ & $\begin{array}{l}269 \\
192 \\
196 \\
386 \\
531 \\
719 \\
837\end{array}$ & $\begin{array}{r}54.6 \\
24.8 \\
24.3 \\
36.7 \\
63.7 \\
88.4 \\
118.9\end{array}$ & $\begin{array}{l}54.6 \\
27.5 \\
29.2 \\
42.2 \\
-69.8 \\
54.7\end{array}$ & $\begin{array}{c}0 \\
-2.7 \\
-4.9 \\
-5.5 \\
\overline{18.6} \\
64.2\end{array}$ \\
\hline $\begin{array}{r}\text { 15GB-A } \\
\mathrm{B} \\
\mathrm{C} \\
\mathrm{D} \\
\mathrm{E} \\
\mathrm{F}\end{array}$ & $\begin{array}{c}\sim 42 \text { wks. } \\
\sigma^{7}\end{array}$ & $\begin{array}{c}\text { Birth } \\
5 \\
31 \\
85 \\
250 \\
389\end{array}$ & $\begin{array}{c}3.77 \\
3.63 \\
4.37 \\
5.9 \\
8.9 \\
10.6\end{array}$ & $\begin{array}{l}55 \\
55 \\
57 \\
62 \\
71 \\
77\end{array}$ & $\begin{array}{l}17.1 \\
20.3 \\
15.7 \\
12.0 \\
13.4 \\
12.1\end{array}$ & $\begin{array}{l}5.1 \\
6.0 \\
5.1 \\
3.5 \\
5.4 \\
5.7\end{array}$ & & $\begin{array}{l}165 \\
186 \\
194 \\
192 \\
118 \\
118\end{array}$ & $\begin{array}{r}100 \\
102 \\
105 \\
103 \\
73 \\
64\end{array}$ & $\begin{array}{l}317 \\
291 \\
339\end{array}$ & $\begin{array}{l}54.2 \\
59.1 \\
53.2 \\
52.6 \\
83.9 \\
89.1\end{array}$ & $\begin{array}{l}54.2 \\
60.3 \\
55.9 \\
53.7 \\
61.2 \\
57.0\end{array}$ & $\begin{array}{c}0 \\
-1.2 \\
-2.7 \\
-1.1 \\
22.7 \\
32.1\end{array}$ \\
\hline $\begin{array}{r}\text { 16GB-A } \\
\text { B } \\
\text { C } \\
\text { D } \\
\text { E } \\
\text { F } \\
\text { G } \\
\text { H } \\
\text { I } \\
\text { J }\end{array}$ & $\begin{array}{c}\sim 40 \text { wks. } \\
\sigma^{T}\end{array}$ & $\begin{array}{c}\text { Birth } \\
7 \\
50 \\
102 \\
185 \\
382 \\
576 \\
718 \\
891 \\
961\end{array}$ & $\begin{array}{c}3.04 \\
2.96 \\
4.43 \\
5.87 \\
7.48 \\
9.8 \\
10.8 \\
11.6 \\
12.5 \\
12.5\end{array}$ & $\begin{array}{l}48 \\
48 \\
56 \\
64 \\
69 \\
76 \\
81 \\
86 \\
90 \\
92\end{array}$ & $\begin{array}{r}18.8 \\
18.1 \\
11.0 \\
11.0 \\
12.2 \\
9.7 \\
11.8 \\
11.3 \\
10.8 \\
10.8\end{array}$ & $\begin{array}{l}5.7 \\
5.1 \\
3.6 \\
3.4 \\
4.0 \\
3.4 \\
3.8 \\
4.0 \\
4.0 \\
3.5\end{array}$ & $\begin{array}{l}62.5 \\
56.3 \\
29.9 \\
32.2 \\
36.9 \\
30 \\
32.6\end{array}$ & $\begin{array}{c}909 \\
93 \\
102 \\
109 \\
86 \\
66 \\
42 \\
35 \\
28.7 \\
32.5\end{array}$ & $\begin{array}{r}100 \\
97 \\
93 \\
106 \\
87 \\
68 \\
39 \\
35 \\
25 \\
33\end{array}$ & & $\begin{array}{r}49.1 \\
39.3 \\
36.9 \\
49.5 \\
67.8 \\
67.8 \\
91.7 \\
95.7 \\
98.9 \\
100.9\end{array}$ & $\begin{array}{l}49.1 \\
38.1 \\
33.9 \\
52.5 \\
58.3 \\
46.1 \\
35.8 \\
33.5 \\
23.7 \\
33.3\end{array}$ & \begin{tabular}{r}
\multicolumn{1}{r}{0} \\
1.2 \\
3.0 \\
-3.0 \\
9.5 \\
21.7 \\
55.9 \\
62.2 \\
75.2 \\
67.6
\end{tabular} \\
\hline $\begin{array}{r}\text { 16GB2-A } \\
\text { B } \\
\mathrm{C} \\
\mathrm{D} \\
\mathrm{E} \\
\mathrm{F} \\
\mathrm{G}\end{array}$ & $\begin{array}{c}40 \text { wks. } \\
\text { Brother } \\
\text { of } 16 \mathrm{~GB} \\
\text { and } \\
16 \mathrm{~GB} 3 \\
\sigma^{7}\end{array}$ & $\begin{array}{c}\text { Birth } \\
7 \\
27 \\
210 \\
293 \\
383 \\
453\end{array}$ & $\begin{array}{l}3.01 \\
3.09 \\
3.57 \\
7.5 \\
8.45 \\
9.2 \\
9.5\end{array}$ & $\begin{array}{l}52 \\
52 \\
56 \\
70 \\
75 \\
78 \\
83\end{array}$ & $\begin{array}{r}21.6 \\
21.0 \\
13.9 \\
10.4 \\
10.4 \\
9.3 \\
9.6\end{array}$ & $\begin{array}{l}5.2 \\
5.4 \\
4.8 \\
4.5 \\
4.3 \\
4.6 \\
5.2\end{array}$ & $\begin{array}{l}64.5 \\
55.7 \\
41.0 \\
33.8 \\
29.7 \\
33.4 \\
32.9\end{array}$ & $\begin{array}{l}326 \\
230 \\
206 \\
216 \\
186\end{array}$ & $\begin{array}{r}100 \\
96 \\
104 \\
81 \\
64 \\
84 \\
69\end{array}$ & $\begin{array}{l}562 \\
641 \\
694 \\
742\end{array}$ & $\begin{array}{l}56.6 \\
51.7 \\
41.0 \\
58.4 \\
66.7 \\
64.5 \\
71.2\end{array}$ & $\begin{array}{l}56.6 \\
49.6 \\
42.6 \\
47.3 \\
42.7 \\
54.2 \\
49.1\end{array}$ & $\begin{array}{c}0 \\
2.1 \\
-1.6 \\
11.1 \\
24.0 \\
10.3 \\
22.1\end{array}$ \\
\hline $\begin{array}{r}16 \mathrm{~GB} 3-\mathrm{A} \\
\mathrm{B} \\
\mathrm{C}\end{array}$ & $\begin{array}{c}\text { 40 wks. } \\
\text { Sister of } \\
16 \mathrm{~GB} \text { and } \\
\text { 16GB2 } \\
\square\end{array}$ & $\begin{array}{c}\text { Birth } \\
5 \\
48\end{array}$ & $\begin{array}{l}3.4 \\
3.18 \\
4.51\end{array}$ & $\begin{array}{l}50 \\
50 \\
58\end{array}$ & $\begin{array}{l}19.3 \\
19.8 \\
10.5\end{array}$ & $\begin{array}{l}4.4 \\
4.6 \\
3.3\end{array}$ & $\begin{array}{l}60.2 \\
57.4 \\
30.6\end{array}$ & $\begin{array}{l}185 \\
203 \\
218\end{array}$ & $\begin{array}{l}100 \\
102 \\
110\end{array}$ & $\begin{array}{l}289 \\
236 \\
353\end{array}$ & $\begin{array}{l}55.8 \\
46.7 \\
37.1\end{array}$ & $\begin{array}{l}55.8 \\
47.6 \\
40.8\end{array}$ & $\begin{array}{c}0 \\
-0.9 \\
-3.7\end{array}$ \\
\hline $\begin{array}{r}\text { 17GB-A } \\
\text { B } \\
\text { C } \\
\text { D }\end{array}$ & $\begin{array}{c}\sim 42 \text { wks. } \\
\sigma^{7}\end{array}$ & $\begin{array}{c}\text { Birth } \\
2 \\
7 \\
363\end{array}$ & $\begin{array}{l}4.39 \\
3.77 \\
4.23 \\
12.2\end{array}$ & $\begin{array}{l}56 \\
56 \\
56 \\
80\end{array}$ & $\begin{array}{l}15.8 \\
16.3 \\
13.7 \\
11.2\end{array}$ & $\begin{array}{l}5.1 \\
5.1 \\
5.0 \\
4.4\end{array}$ & $\begin{array}{l}49 \\
49.4 \\
46.4 \\
36.1\end{array}$ & $\begin{array}{l}82 \\
86 \\
85 \\
62\end{array}$ & $\begin{array}{r}100 \\
103 \\
113 \\
79\end{array}$ & $\begin{array}{l}352 \\
304 \\
328 \\
818\end{array}$ & $\begin{array}{l}55.6 \\
49.6 \\
44.9 \\
91.6\end{array}$ & $\begin{array}{l}55.6 \\
51.1 \\
51.2 \\
72.4\end{array}$ & $\begin{array}{c}0 \\
-1.5 \\
-6.3 \\
19.2\end{array}$ \\
\hline
\end{tabular}

T Sample lost in laboratory; $\mathrm{cpm} / \mathrm{ml}$. assumed on basis of 7-day sample and trend in counts for other infants. 


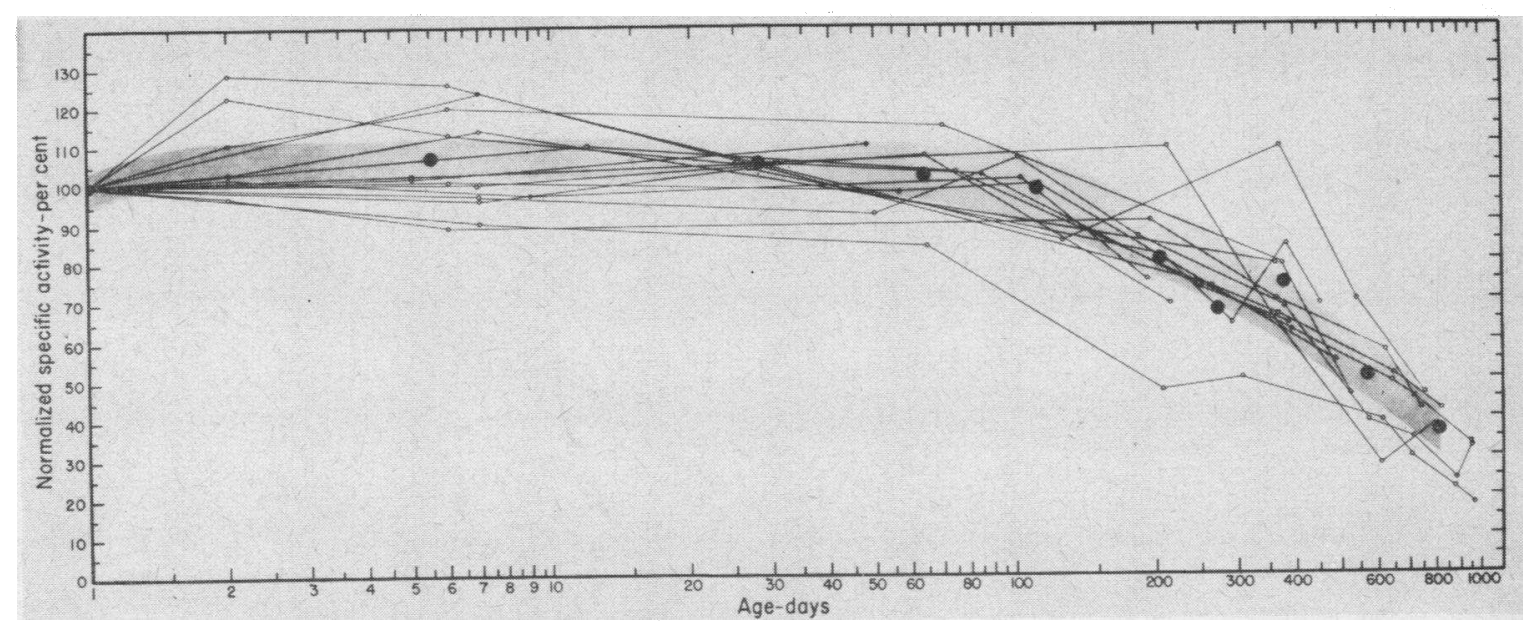

Fig. 2. Normalized Specific Activities of 16 Infants up to 32 Months after Birth

The lines connect consecutive data for each individual infant. The black dots (unconnected) indicate average values for the means of the following age groups:
1. Birth
2. 2-12 days
6. 151-216 days
3. $13-31$ days
7. 217-309 days
4. 32-100 days
8. $310-400$ days
5. 101-150 days
9. 401-675 days
10. 676-973 days

The shaded section indicates the area bounded by 2 standard errors above and below the best curve through the average values.

The average normalized specific activities above 100 per cent are interpreted as resulting from circumstances of maternal $\mathrm{Fe}^{55}$ administration (see text).

as birth approached. As a result, hemoglobin in fetal erythrocytes made shortly before birth must have contained more $\mathrm{Fe}^{55}$ than hemoglobin in cells of earlier manufacture. Survival of these younger cells after degeneration of the older ones would produce the effect observed. Blood from the five infants born after the later pregnancies during which no $\mathrm{Fe}^{55}$ was introduced showed little or no specific activity rise in the early post-natal period.

The relatively constant level maintained by the specific activities plotted in Figure 2 until approximately 100 days after birth indicates no appreciable use of food iron for hemoglobin formation during this time. Thereafter, dietary iron appears in the circulating hemoglobin in progressively increasing amounts. At 800 days (26 months) for example, approximately 20 to 50 per cent of the hemoglobin is still formed from transplacental iron, the remaining 50 to 80 per cent from dietary iron.

A statement of the actual amounts of transplacental and dietary iron in the total circulating hemoglobin requires knowledge of blood volume. This could not be directly obtained but an approxi- mate value was calculated from physical measurements of each infant at each sampling. The formulae used for derivation of blood volumes according to the data of other investigators (7-9) are described in the note under Table $I$, and the calculated blood volumes are shown in Column 11 of that table.

The total amounts of circulating hemoglobin, computed from calculated blood volumes and measured hemoglobin concentrations, are listed in Column 12 of Table I and plotted in Figure 3. The mean total circulating hemoglobin at 2 to 3 months of age was approximately equal to the mean at birth; the mean at $1 \frac{1}{2}$ to 2 years of age was twice that at birth.

The amounts of hemoglobin iron of transplacental and dietary origin, calculated from specific activity measurements, are listed in Table $\mathrm{I}$ and shown graphically in Figures 4A and 4B. Figure 4A indicates that the mean amount of hemoglobin made from transplacental iron remains essentially constant throughout the period of these investigations. However, the elevation of the mean by some 20 per cent above the birth level 


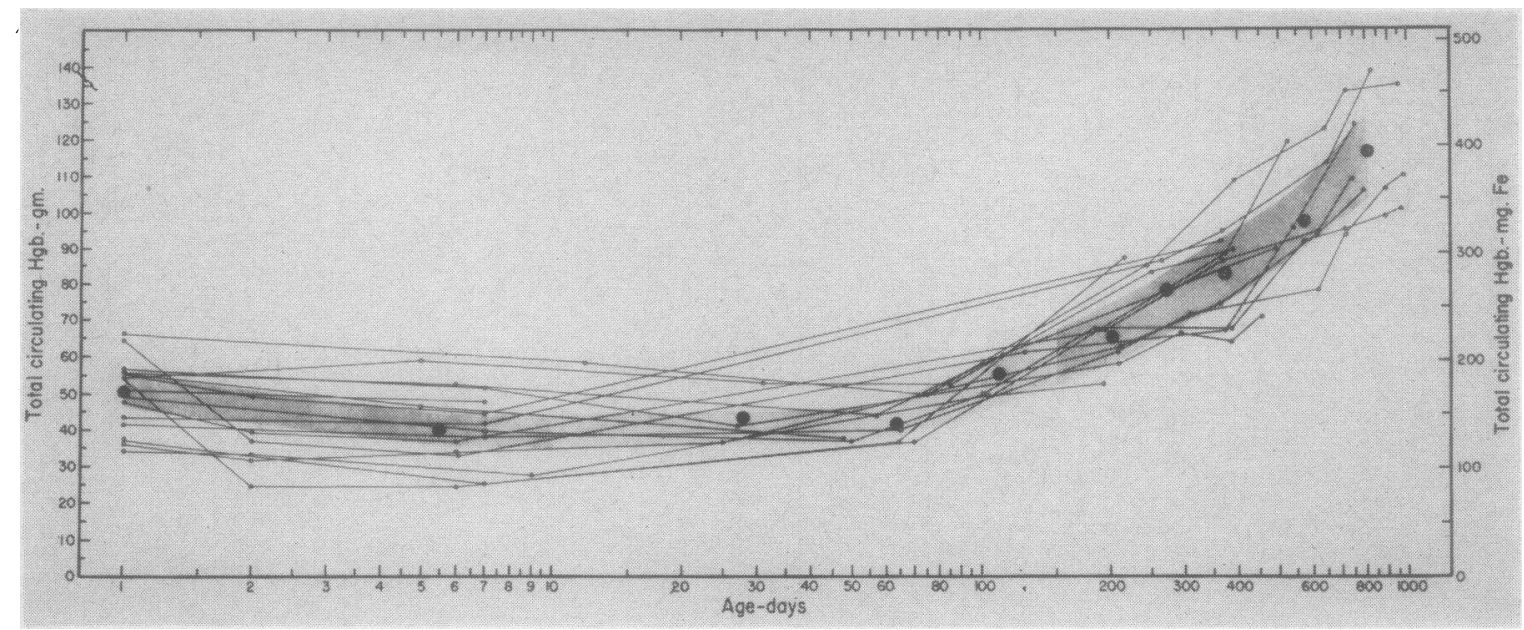

Fig. 3. Total Circulating Hemoglobin of 16 Infants up to 32 Months after Birth (For details of plotting see Figure 2.)

at approximately 200 to 400 days suggests hemoglobin formation from transplacental iron in addition to that which was in hemoglobin at birth. In the following discussion, such iron will be referred to as "stored" transplacental iron even though hemoglobin at birth is also in a sense a storage depot. One cannot assume, in view of the wide statistical confidence limits of the data, that stored transplacental iron regularly represents 20 per cent more than that in hemoglobin at birth;

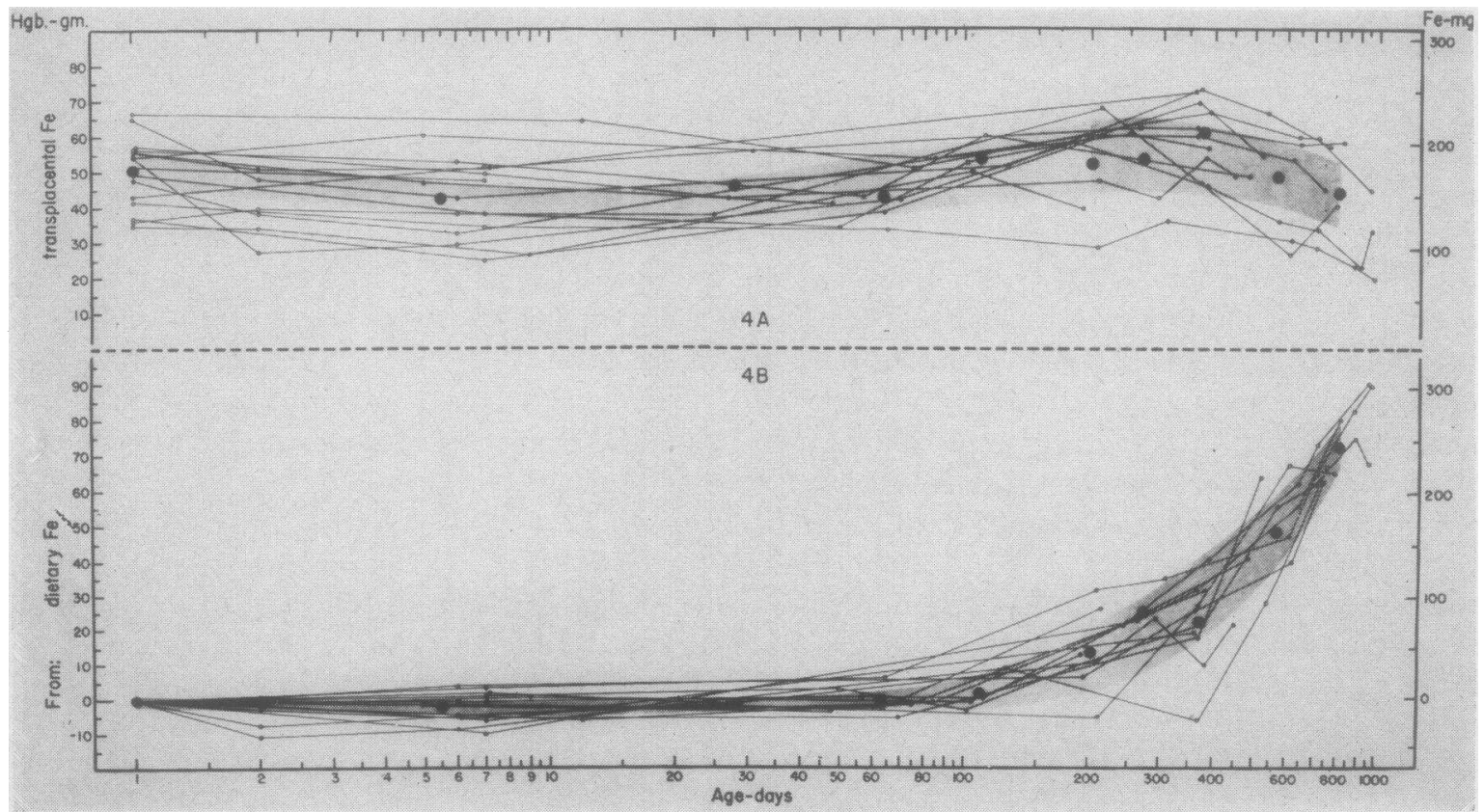

Fig. 4A. Hemoglobin from Transplacental Iron of 16 Infants up to 32 Months after Birth

Fig. 4B. Hemoglobin from Dietary Iron of 16 Infants up to 32 Months after Birth

Negative values for individual and average hemoglobins from dietary iron may arise partly from statistical considerations and partly from the fact that some of the normalized specific activities of Figure 2 are greater than 100 per cent.

(For details of plotting see Figure 2.) 
nevertheless, the findings suggest that at least some iron is available to the infant from non-hemoglobin stores.

Similarly, the data as a whole do not allow the conclusion that the terminal downward trend of the mean in Figure 4A definitely indicates a loss of transplacental iron from the blood, even though such a loss appears to have occurred in individual infants, as will be discussed below. The decline of specific activities shown in Figure 2 results primarily from dilution with dietary iron. Figure 4B indicates the average amount of hemoglobin made from iron obtained from the diet: essentially none before the 4 th month, ${ }^{5}$ about 10 grams by 6 months, 22 grams by 1 year, and 65 grams by 2 years.

\section{DISCUSSION}

Direct measurement of iron intake and loss was not attempted in these studies. Relatively little information has been found in the literature regarding the amount of iron normally absorbed and excreted by infants, particularly during the first few months after birth. Oettinger, Mills, and Hahn (10) have demonstrated that tagged iron administered by stomach tube is absorbed by term and premature infants at a few days of age. At least under the experimental conditions of that study, the rate of incorporation of iron into hemoglobin is extremely small $(0.01 \mu \mathrm{g}$. Fe per day) compared to total circulating hemoglobin (150 $\mathrm{mg}$. Fe). Incorporation of $0.01 \mu \mathrm{g}$. Fe per day would not have been detected by the methods of the present study. Oettinger, Mills, and Hahn (10) gave no data regarding the excretion of the test iron. Balance studies by other workers (1) indicate that infants of less than 3 months are in net iron equilibrium on a daily intake of about 0.15 mg. per $\mathrm{Kg}$., and in negative balance on smaller iron intakes. Such figures do not differentiate between iron which fails to be absorbed and that which is excreted from endogenous sources into the bowel, except as excretion may be inferred from the negative balances when the diet contains

\footnotetext{
5 Assuming constant daily incorporation of dietary iron into hemoglobin resulting in a significant decrease in the specific activity at 4 months, the rate of incorporation would have to be at least $\sim 50 \mu \mathrm{g}$. Fe per infant per day to be detectable at this time.
}

less than $0.15 \mathrm{mg}$. per $\mathrm{Kg}$. If the infant's endogenous iron loss is actually $0.15 \mathrm{mg}$. per $\mathrm{Kg}$. per day, that amount would be disproportionately large compared to the total daily iron loss of 1.0 mg. usually assumed for the $70 \mathrm{Kg}$. adult.

Thus while it has been demonstrated by others that the infant may both absorb and excrete iron, the amounts have not been accurately determined. The present investigation suggests absorption and loss of much less iron than is implied by available balance data. In the infants of this study, as a group, there was no indication of either absorption or loss during a period extending into the fourth month after birth. The decline of hematopoiesis (11) and reduction in mean red blood cell size and mean corpuscular hemoglobin (12) normally occurring in this age period, together with dilution by body growth and blood volume expansion, produce the fall in hemoglobin concentration apparent in Figure 1. The total amounts of hemoglobin of the infants studied are shown by the mean in Figure 3 to have remained fairly steady until a rising trend appeared at about 60 days. Thus, the amount of hemoglobin began to increase 1 to 2 months before there was evidence of any dietary iron in the blood (Figure 4B). Transplacental iron was apparently sufficient for the beginning expansion of circulating hemoglobin.

Between the third or fourth month and the end of the second year of age, the concentration of hemoglobin increased only slightly, while the total amount of hemoglobin was doubled, primarily by addition of that made from dietary iron. The data presented in Figure $4 \mathrm{~A}$ indicate that little of the original hemoglobin iron left the blood during this period, whereas some transplacental iron from stores may have participated in the increase in hemoglobin.

The amounts of transplacental iron apparently emerging from "stores" varied in the individual infants. It might be expected that the total amount of iron in hemoglobin at birth would parallel the amount stored elsewhere and that an infant born with a relatively low total circulating hemoglobin would have comparatively little stored iron for later blood formation. To test this possibility, the largest addition of hemoglobin from stored iron for each of the 14 infants studied for more than 100 days was plotted against the total hemoglobin of that infant at birth (Figure 5). 
Except for one instance (Infant 5GB), the data suggest that infants with low total hemoglobin at birth seemed to have relatively large amounts of stored iron available for later hemoglobin manufacture.

It cannot be argued that those infants born with much circulating hemoglobin were necessarily lacking in iron stored elsewhere, since they may not have been required to draw upon such iron later. Nevertheless, it is apparent that the fullterm infant's iron resources cannot be predicted from his hemoglobin concentration and body size at birth. Some prediction of low fetal storage can be made when the maternal hemoglobin concentration in late pregnancy is pathologically low, as shown by the work of Strauss (13). But Woodruff and Bridgeforth (14) found no such correlation when the hemoglobin concentration of the mother's blood remains above 9 grams per cent. In the present investigation, the hemoglobin of one mother fell to 10.2 grams per cent; all others had more than 11 grams per cent hemoglobin during the last trimester of their pregnancies.

Data from infants followed sufficiently long indicate that dietary iron not only continues to be added to transplacental iron but gradually begins to replace it in hemoglobin formation during the third year, as is suggested by the late downward trend of the mean in Figure 4A.

The preceding discussion applies to the general pattern of iron utilization in the infants as a group. Variations shown by certain individuals deserve brief presentation. Perhaps the most interesting was Infant 5GB. While not premature by the usual standards of weight, this infant was born one month before expected date of confinement. The evidence obtained from ten blood samples over a period of 973 days is shown in Figure 6. The infant, a Negro male, gained weight slowly after two months of rapid growth. In spite of two rather severe infections he had no definite anemia, though medicinal iron (ferrous sulfate, $240 \mathrm{mg}$. per day) was prescribed for 6 weeks in his second year when his hemoglobin was found to be 11.2 grams per cent. His mother's dietary intake of iron during pregnancy was rated as very poor; his own diet was relatively high in iron during the first six months and then somewhat lower but still adequate.

Because of his small size this infant's total circulating hemoglobin was only 42 grams at birth. As Figure 6 shows, dietary iron appeared in his hemoglobin by 65 days after birth. This process continued so consistently that by 2 years only 30 per cent of the iron in his hemoglobin was of trans-

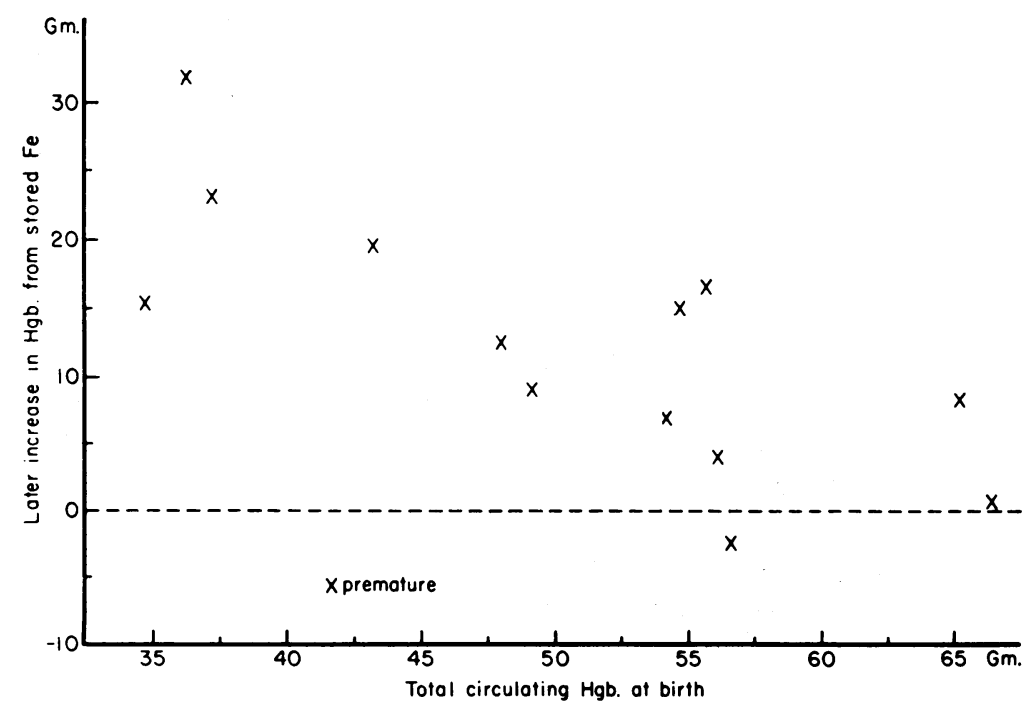

Fig. 5. Relation between Maximum Emergence of Transplacental Iron after Birth and Total. Circulating Hemoglobin at Birth

(Fourteen infants; omitting Infants $12 \mathrm{~GB} 2$ and 16GB3, studied less than 100 days.) 


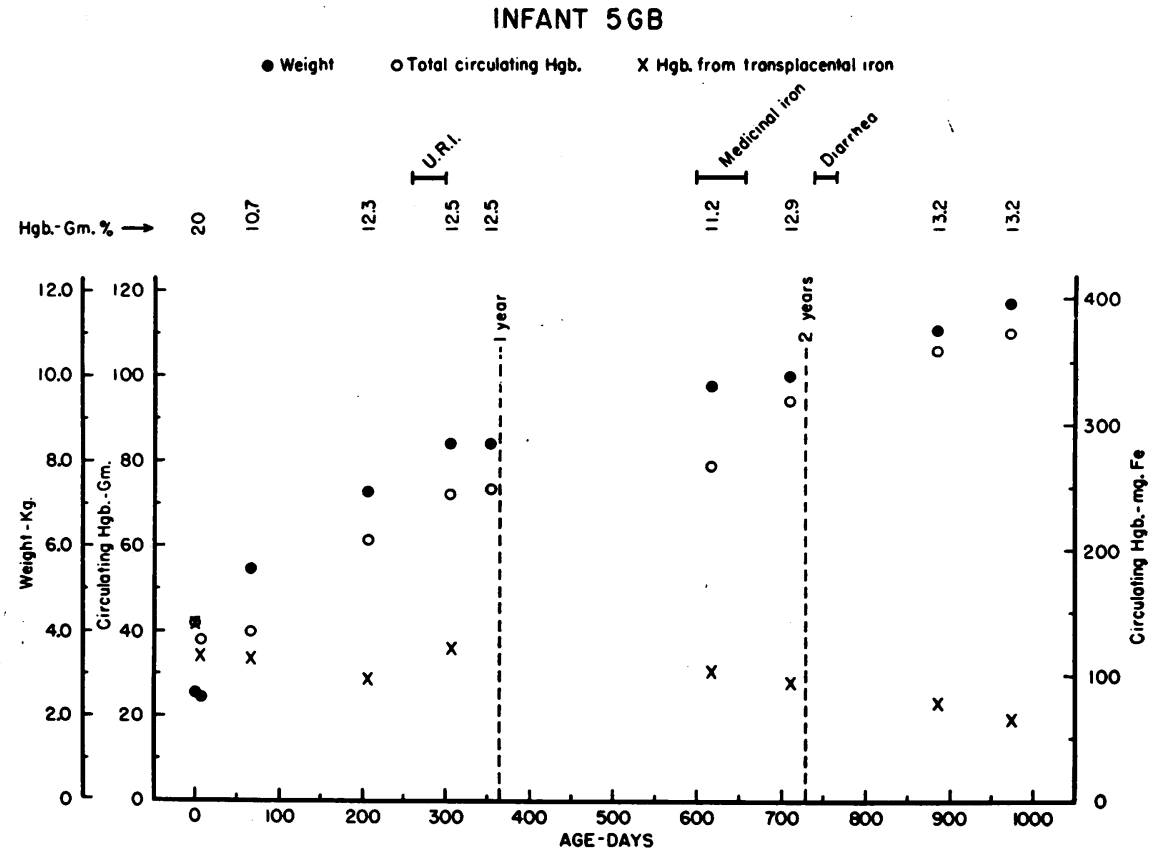

Fig. 6. Iron Utilization for Hemoglobin Formation in a 4-Week Premature, 2.53 Kg. Infant; Birth to 32 Months of AgE

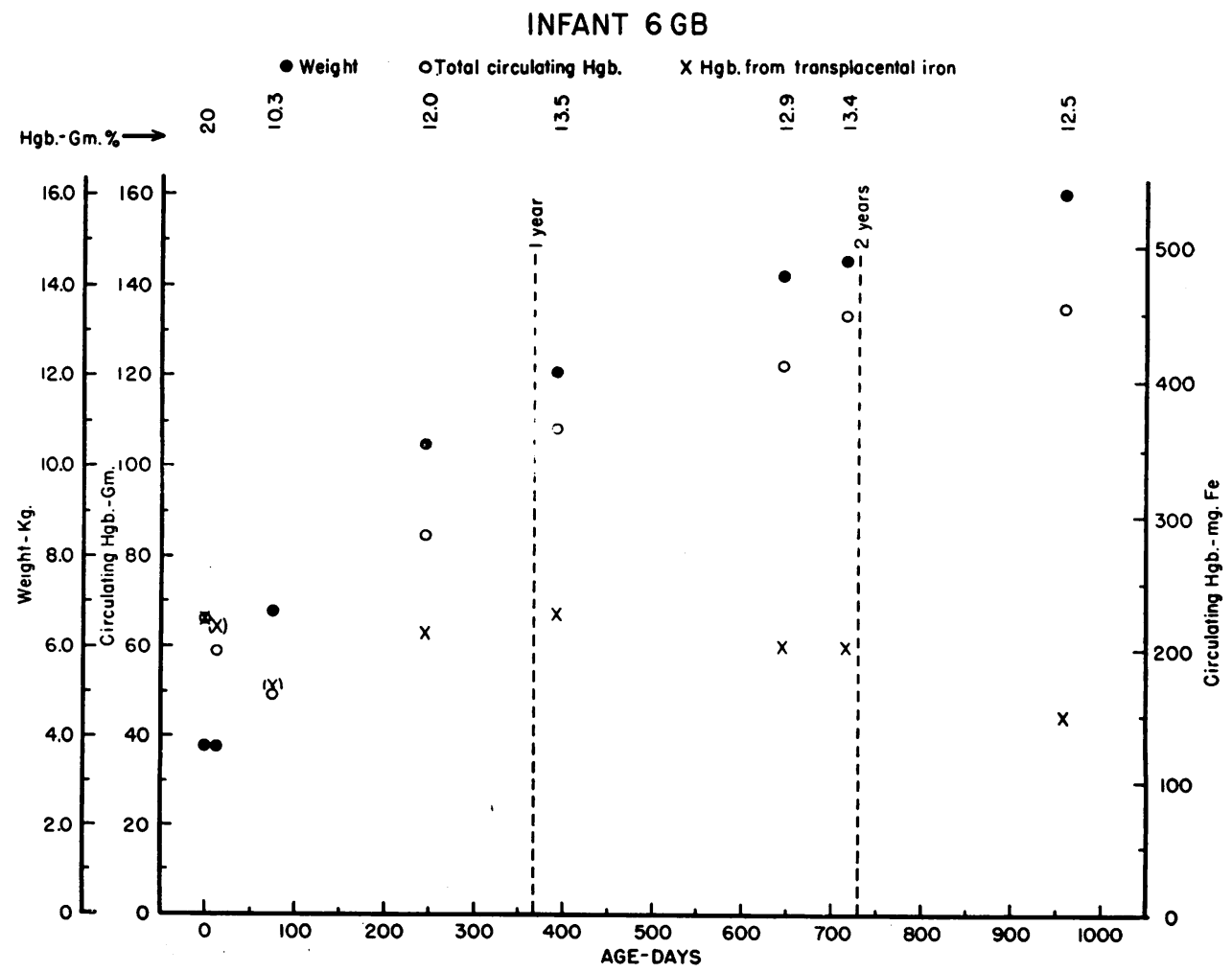

Fig. 7. Iron Utilization for Hemoglobin Formation in a 3.8 Kg. Infant; Birth to 32 Months of AGE

Values of hemoglobin from transplacental iron greater than the total circulating hemoglobin (indicated by parenthetic symbols) arise from normalized specific activities above 100 per cent, discussed in text. 
placental origin. At no time was there evidence of stored iron appearing in blood formation. The provision of medicinal iron at 600 to 640 days was followed by a slight increase in hemoglobin concentration and in total circulating hemoglobin. The data suggest that the increased hemoglobin was not manufactured from transplacental iron.

In summary, this infant, born prematurely to a poorly nourished mother, was outstanding among the group by virtue of his low total hemoglobin at birth and the consistently small contribution of transplacental iron to later hemoglobin formation. Both of these features might well be consequences of prematurity. About $70 \mathrm{mg}$. of iron originally present had disappeared from this infant's blood at 32 months. Although not excessive in amount when compared to the trend of mean values, 70 $\mathrm{mg}$. of iron represented an inordinately large proportion of that acquired by this infant during fetal life. The data further suggest that iron added to this infant's oral intake was utilized for hemoglobin formation.

Infant $6 \mathrm{~GB}$ (Figure 7 ) affords an interesting comparison. His mother had been well-nourished before and during pregnancy. His own diet was excellent and he remained free of infections. Although born 13 days before the expected date, his birth weight of $3.8 \mathrm{Kg}$. argues against any significant prematurity. He gained about 30 per cent more weight than the infant just considered during the 32 months over which both were followed.

The iron metabolism of this infant and that of the premature one just discussed are similar in many respects. Their hemoglobin concentrations were similar at birth and thereafter. Neither showed evidence of any significant utilization of stored transplacental iron. Both formed approximately the same amounts of hemoglobin from dietary iron at comparable ages. Finally, about the same amount of transplacental iron (70 mg.) had apparently disappeared from the blood of both at 32 months of age. However, Infant 6GB was not only larger at birth but gained more weight thereafter. As a result, he consistently maintained a higher total of circulating hemoglobin, a relatively larger proportion of which was

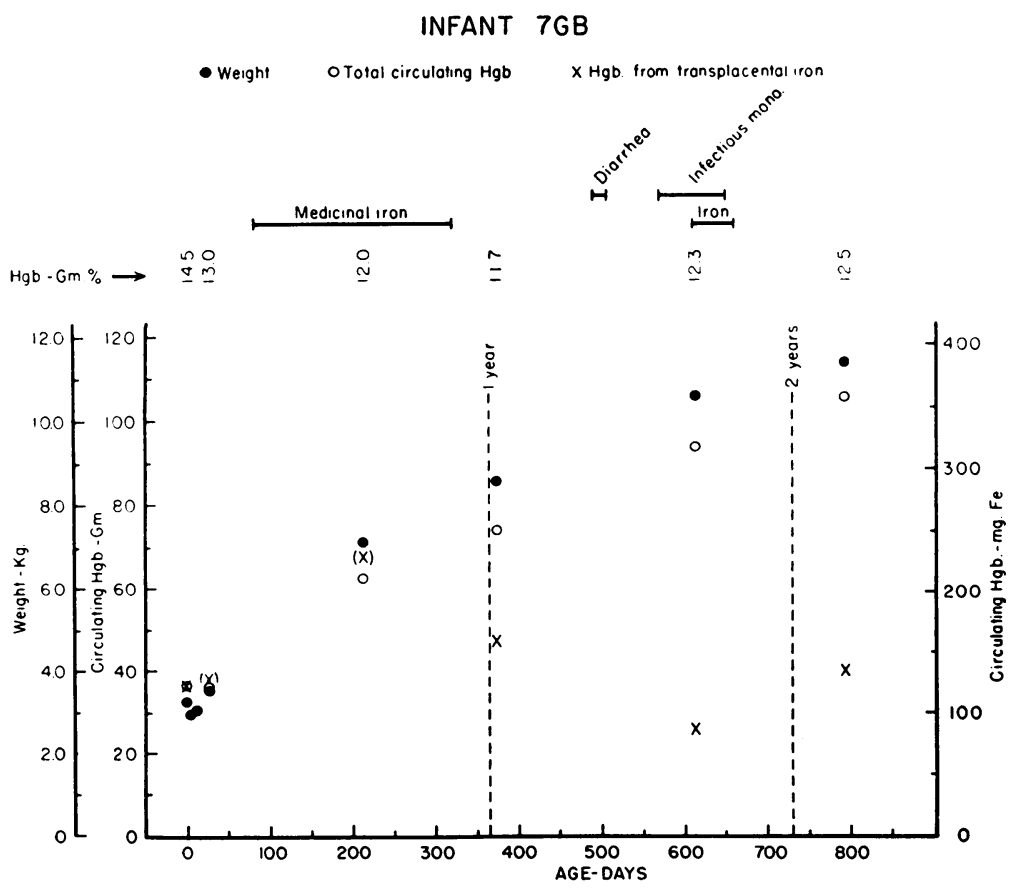

Fig. 8. Iron Utilization in a $3.24 \mathrm{Kg}$. Infant Born with Low Total Circulating Hemoglobin; Birth to 26 Months of Age

Data show emergence of transplacental iron from stores during first 200 days, and suggest similar emergence between 600 and 800 days. For comment on parenthetic symbols, see legend of Figure 7. 
made from the transplacental iron present in his hemoglobin at birth. It is perhaps unfortunate that this infant had so good a diet. Otherwise his resources of stored transplacental iron might have become apparent. As they stand, his data indicate the large contribution which may be made by the hemoglobin at birth to iron needs throughout infancy.

In contrast to the two infants just discussed, the data from Infant 7GB (Figure 8) indicated the emergence of considerable transplacental iron from stores. This infant was born 9 days after expected confinement to a mother whose diet had been good. Birth weight was $3.24 \mathrm{Kg}$. The hemoglobin concentration of $14.6 \mathrm{gm}$. per cent in cord blood was low, without apparent reason from the obstetrical history or studies made during this mother's pregnancy (3). The similarity of hemoglobin concentrations in samples taken at 2 and 6 days after birth substantiates the measurement from cord blood.

The infant was fed from the breast for 120 days, and thereafter artificially but on an adequate diet. For unknown reasons, the family physician prescribed ferrous sulfate in dosages from 40 to 160 mg. daily for most of the first year. The data of Figure 8 suggest that none of this iron was used for hemoglobin during the first 7 months, whereas about $100 \mathrm{mg}$. of transplacental iron, which could not have been in the blood at birth, apparently entered the circulating hemoglobin by 200 days. Similar emergence of iron from stores in other infants born with low concentrations or total amounts of hemoglobin has been commented upon above. From 200 to 600 days, transplacental iron in hemoglobin decreased to below the birth level. At some time between 600 and 800 days after birth, and following a moderate attack of infectious mononucleosis, a second emergence of transplacental iron is suggested by the data. This infant's blood showed approximately the same calculated amount of transplacental iron at 26 months of age as was in her hemoglobin at birth. It is difficult to relate the two periods of medicinal iron administration to the appearance of iron from storage in this child's course.

\section{SUMMARY}

Sixteen infants of mothers who had received transfusions of red blood cells containing $\mathrm{Fe}^{55}$ were studied for periods up to 32 months of age. Measurements of R.B.C., Hgb., Hct., total iron and $\mathrm{Fe}^{55}$ of erythrocytes, were made from umbilical cord blood and from venous blood obtained at intervals during the study period.

Results from the 16 infants as a group indicated very little or no utilization of dietary iron for hemoglobin until 3 to 4 months after birth. At 1 year, iron of transplacental origin constituted $\sim 70$ per cent of total hemoglobin iron; at 2 years, $\sim 40$ per cent. In absolute terms these values correspond to $\sim 170 \mathrm{mg}$. transplacental iron at birth, $\sim 200 \mathrm{mg}$. at 1 year, and $\sim 160 \mathrm{mg}$. at 2 years in the total circulating hemoglobin. At 2 years, over 90 per cent of transplacental iron was still utilized for hemoglobin.

A 20 per cent rise in transplacental iron at 200 to 400 days over that present in hemoglobin at birth suggested utilization of iron stored elsewhere during fetal life. Infants with relatively low total circulating hemoglobin at birth tended to show relatively large amounts of such stored iron in later hemoglobin manufacture.

Individual infants deviated from the pattern described for the group. In particular, a premature infant of 36 weeks' gestation had low total circulating hemoglobin at birth, no evidence of any stored iron in later hemoglobin formation and proportionately high utilization of dietary iron, which first appeared in hemoglobin at 65 days after birth.

It is concluded that the hemoglobin of normal infants (A) utilizes iron obtained during fetal life throughout infancy, (B) incorporates significant amounts of dietary iron only after 3 or 4 months of age, and (C) by two years reaches a total of $\sim 115$ grams, composed of $\sim 70$ grams from dietary and $\sim 45$ from transplacental iron. Prematurity increases the proportionate contribution of dietary iron.

\section{ACKNOWLEDGMENTS}

The authors are grateful to Doctors Robley Evans, Petter Karlberg, Louis Diamond, and Randall Caswell for advice and assistance; to Doctors Myron Feinstein, Kenneth Phillips, and Robert Roth for obtaining blood specimens; to Mrs. Bertha Burke for obtaining and evaluating nutrition histories, and to the Hematology Laboratory of the Children's Hospital for blood counts and hemoglobin measurements. 


\section{REFERENCES}

1. Josephs, H. W., Iron metabolism and the hypochromic anemia of infancy. Medicine, 1953, 32, 125.

2. Caton, W. L., Roby, C. C., Reid, D. E., and Gibson, J. G., 2nd, Plasma volume and extravascular fluid volume during pregnancy and the puerperium. Am. J. Obst. \& Gynec., 1949, 57, 471.

3. Caton, W. L., Roby, C. C., Reid, D. E., Caswell, R., Maletskos, C. J., Fluharty, R. G., and Gibson, J. G., 2nd, The circulating red cell volume and body hematocrit in normal pregnancy and the puerperium. Am. J. Obst. \& Gynec., 1951, 61, 1207.

4. Diamond, L. K., Smith, N. J., and Vaughan, V. C., Diseases of the blood in Textbook of Pediatrics, W. E. Nelson, Ed., 6th Edition, Philadelphia, W. B. Saunders Co., 1954, pp. 949-1014.

5. Peacock, W. C., Evans, R. D., Irvine, J. W., Jr., Good, W. M., Kip, A. F., Weiss; S., and Gibson, J. G., 2nd, The use of two radioactive isotopes of iron in tracer studies of erythrocytes. J. Clin. Invest., 1946, 25, 605.

6. Du Bois, D., and Du Bois, E. F., Clinical calorimetry. $\mathrm{X}$. A formula to estimate the approximate surface area if height and weight be known. Arch. Int. Med., 1916, 17, 863.
7. Mollison, P. L., Veall, N., and Cutbush, M., Red cell and plasma volume in newborn infants. Arch. Dis. Childhood, 1950, 25, 242.

8. Russell, S. J. M., Blood volume studies in healthy children. Arch. Dis. Childhood, 1949, 24, 88.

9. Karlberg, P., and Lind, J., Studies of the total amount of hemoglobin and the blood volume in children. I. Determination of total hemoglobin and blood volume in normal children. Acta paediat., 1955, 44, 17.

10. Oettinger, L., Jr., Mills, W. B., and Hahn, P. F., Iron absorption in premature and full-term infants. J. Pediat., 1954, 45, 302.

11. Faxen, N., Red blood picture in healthy infants. Acta paediat., 1937, 19, (Suppl. 1).

12. Guest, G. M., Brown, E. W., and Wing, M., Erythrocytes and hemoglobin of blood in infancy and in childhood. II. Variability in number, size and hemoglobin content of the erythrocytes during the first five years of life. Am. J. Dis. Child., 1938, $56,529$.

13. Strauss, M. B., Anemia of infancy from maternal iron deficiency in pregnancy. J. Clin. Invest., 1933, 12, 345.

14. Woodruff, C. W., and Bridgeforth, E. B., Relationship between the hemogram of the infant and that of the mother during pregnancy. Pediatrics, $1953,12,681$. 